\title{
Space and time dimensions of algebras with application to Lorentzian noncommutative geometry and quantum electrodynamics
}

Nadir Bizi, Christian Brouder, and Fabien Besnard

Citation: Journal of Mathematical Physics 59, 062303 (2018); doi: 10.1063/1.5010424

View online: https://doi.org/10.1063/1.5010424

View Table of Contents: http://aip.scitation.org/toc/jmp/59/6

Published by the American Institute of Physics

\section{Articles you may be interested in}

Families of spectral triples and foliations of space(time)

Journal of Mathematical Physics 59, 063507 (2018); 10.1063/1.5021305

Series solutions of Laguerre- and Jacobi-type differential equations in terms of orthogonal polynomials and physical applications

Journal of Mathematical Physics 59, 063508 (2018); 10.1063/1.5027158

From phase space to multivector matrix models

Journal of Mathematical Physics 59, 062302 (2018); 10.1063/1.4986228

Framed M-branes, corners, and topological invariants

Journal of Mathematical Physics 59, 062304 (2018); 10.1063/1.5007185

$\mathrm{SL}(3, \mathrm{C})$ structure of one-dimensional Schrödinger equation

Journal of Mathematical Physics 59, 062106 (2018); 10.1063/1.4994125

Generalized Fock spaces and the Stirling numbers

Journal of Mathematical Physics 59, 063509 (2018); 10.1063/1.5035352

\section{PHYSICS TODAY}

WHITEPAPERS
MANAGER'S GUIDE

Accelerate R\&D with

Multiphysics Simulation
READ NOW

PRESENTED BY

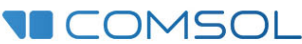




\title{
Space and time dimensions of algebras with application to Lorentzian noncommutative geometry and quantum electrodynamics
}

\author{
Nadir Bizi, ${ }^{1}$ Christian Brouder, ${ }^{1, a)}$ and Fabien Besnard ${ }^{2}$ \\ ${ }^{1}$ Sorbonne Université, UPMC Univ. Paris 06, UMR CNRS 7590, Institut de Minéralogie, \\ de Physique des Matériaux et de Cosmochimie, Muséum National d'Histoire Naturelle, \\ IRD UMR 206, 4 place Jussieu, F-75005 Paris, France \\ ${ }^{2}$ Pôle de recherche M.L. Paris, EPF, 3 bis rue Lakanal, F-92330 Sceaux, France
}

(Received 25 October 2017; accepted 24 May 2018; published online 19 June 2018)

\begin{abstract}
An analogy with real Clifford algebras on even-dimensional vector spaces suggests assigning an ordered pair $(s, t)$ of space and time dimensions (or equivalently an ordered pair $(m, n)$ of metric and $K O$ dimensions) modulo 8 to any algebraic structure (that we call $C P T$ corepresentation) represented over a Hilbert space by two selfadjoint involutions and an anti-unitary operator having specific commutation relations. It is shown that this assignment is compatible with the tensor product: the space and time dimensions of the tensor product of two $C P T$ corepresentations are the sums of the space and time dimensions of its factors, and the same holds for the metric and $K O$ dimensions. This could provide an interpretation of the presence of such algebras in $P T$-symmetric Hamiltonians or the description of topological matter. This construction is used to build an indefinite (i.e., pseudo-Riemannian) version of the spectral triple of noncommutative geometry, defined over a Krein space and classified by the pair $(m, n)$ instead of the $K O$ dimension only. Within this framework, we can express the Lagrangian (both bosonic and fermionic) of a Lorentzian almost-commutative spectral triple. We exhibit a space of physical states that solves the fermion-doubling problem. The example of quantum electrodynamics is described. Published by AIP Publishing. https://doi.org/10.1063/1.5010424
\end{abstract}

\section{INTRODUCTION}

Clifford algebras are at the heart of the description of matter not only because fermions (spinors) are their irreducible representations, but also because they classify topological insulators and superconductors. ${ }^{1,2}$ They are also used as a template for deeper structures, such as $K$-theory ${ }^{3,4}$ or noncommutative geometry (NCG) ${ }^{5}$ which pervade physics from topological matter to disordered systems and the standard model of particles.

The main aim of this paper is to describe a pseudo-Riemannian analog of noncommutative geometry, but on the way we put forward a procedure to assign a space dimension and a time dimension to some algebraic structure that we define now. We need the following:

- A complex Hilbert space $\mathcal{H}$.

- A self-adjoint involution $\chi$ (i.e., $\chi^{2}=1$ ) defining the parity of operators: an operator $a$ on $\mathcal{H}$ is even if $\chi a \chi=a$ and odd if $\chi a \chi=-a$. For example, $\chi$ can be the chirality operator or the inversion symmetry.

- A second self-adjoint involution $\eta$, which can be the flat-band Hamiltonian sign $H^{6}$ or a fundamental symmetry.

- An anti-linear (charge conjugation) map $J$ such that $J^{\dagger} J=1$ and $J^{2}=\epsilon= \pm 1$.

\footnotetext{
a)Electronic mail: christian.brouder@upmc.fr
} 
- Specific commutation or anticommutation relations between $\chi, \eta$, and $J$ defined by three signs $\left(\epsilon^{\prime \prime}, \kappa, \kappa^{\prime \prime}\right)$ introduced in Eqs. (3)-(5).

In a Clifford algebra $C \ell(p, q)$ such that $p+q$ is even, the four signs $\left(\epsilon, \epsilon^{\prime \prime}, \kappa, \kappa^{\prime \prime}\right)$ determine a pair of space and time dimensions $(s, t)$ modulo 8 in a unique way. We propose to assign the same dimensions $(s, t)$ to any algebra satisfying the same relations between $\chi, \eta$, and $J$. This is similar to the way Atiyah related the $K O$-dimension to $p-q \bmod 8$ in Clifford algebras. ${ }^{3}$ These two dimensions solve the question whether indefinite spectral triples have a notion corresponding to the KO-dimension. ${ }^{7}$

Such an assignment is meaningful because it is compatible with the tensor product: the dimensions corresponding to the signs of the (graded) tensor product $A_{1} \hat{\otimes} A_{2}$ of two such algebras are the sum of the dimensions of $A_{1}$ and $A_{2} \bmod 8$.

When we apply this construction to a spectral triple of noncommutative geometry, $\chi$ is the usual chirality operator, $\eta$ is a fundamental symmetry defining a Krein-space structure on $\mathcal{H}$, and $J$ is the usual charge conjugation. In the case of an almost-commutative spectral triple, the space and time dimensions of the commutative (manifold) triple are the physical ones and space and time dimensions are also assigned to the finite triple.

The paper starts with a description of $\chi, \eta$, and $J$ in a Clifford algebra, which sets up the correspondence between commutation relations and space-time dimensions. Then, this correspondence is shown to hold for more general algebras by proving that it is compatible with the graded tensor product of algebras. In Sec. IV, we introduce Krein spaces, which are the natural generalizations of Hilbert spaces associated with spinors on pseudo-Riemannian manifolds. Section V defines the corresponding generalized spectral triples, which we call indefinite spectral triples. This framework is then applied to define the spectral triple of Lorentzian quantum electrodynamics (QED) and its Lagrangian.

\section{AUTOMORPHISMS OF CLIFFORD ALGEBRAS}

We investigate the commutation relations of three operators in Clifford algebras over vector spaces of even dimension $2 \ell$. They are simple algebras whose irreducible representation is $S \simeq \mathbb{C}^{2^{\ell}}$.

The Clifford algebra $C \ell(p, q)$, with $p+q=2 \ell$, is the real generated by $2 \ell$ matrices $\gamma_{j}$ over $S$ such that $\gamma_{i} \gamma_{j}+\gamma_{j} \gamma_{i}=2 \epsilon_{j} \delta_{i j}$, where $p$ coefficients $\epsilon_{j}$ are equal to +1 and $q$ are equal to -1 .

An indefinite inner product on a complex vector space $V$ is a non-degenerate Hermitian form (i.e., a sesquilinear form on $V$ which satisfies $(v, u)=\overline{(u, v)}$ ). It is indefinite because we do not assume that $(v, v)>0$ if $v \neq 0$. It was shown ${ }^{8-10}$ that $S$ can be equipped with two indefinite inner products $(\cdot, \cdot)_{+}$and $(\cdot, \cdot)_{-}$such that

$$
\left(\gamma_{j} \phi, \psi\right)_{ \pm}= \pm\left(\phi, \gamma_{j} \psi\right)_{ \pm}
$$

for every $\gamma_{j}$, and every elements $\phi$ and $\psi$ of $S$. These two inner products are unique up to multiplication by a real factor, and they are invariant under the action of $\operatorname{Spin}(p, q)^{+}$, the connected component of the identity in the spin group $\operatorname{Spin}(p, q)$. Moreover, $S$ can be equipped with two charge conjugations $J_{ \pm}$, which are anti-linear maps such that $J_{ \pm} \gamma_{j}= \pm \gamma_{j} J_{ \pm}$.

To give a concrete representation of the indefinite inner products and the charge conjugations, we equip the complex vector space $S$ with its standard (positive definite) scalar product $\langle\cdot, \cdot\rangle$. By using results scattered in the literature, ${ }^{8,11-15}$ we can state the following. In all representations used in practice, the gamma matrices satisfy $\gamma_{j}^{\dagger}=\epsilon_{j} \gamma_{j}=\gamma_{j}^{-1}$, where $\dagger$ denotes the adjoint with respect to the scalar product, and $\overline{\gamma_{j}}=\zeta_{j} \gamma_{j}$, where the overline denotes the complex conjugation and $\zeta_{j}$ is +1 ( $\gamma_{j}$ is real) or -1 ( $\gamma_{j}$ is imaginary). Shirokov showed ${ }^{15}$ that these representations can be classified by the number of their symmetric gamma matrices (i.e., such that $\gamma_{j}^{T}=\gamma_{j}$ ) modulo 4 , which is equal to $\ell+\zeta \bmod 4$, where $\zeta$ can take the value 0 or 1 . Both cases are useful in practice. For example, the most common representations of $C \ell(1,3)$ are the Dirac, Majorana, and chiral gamma matrices. ${ }^{16}$ The number of symmetric matrices of these representations is 2,3 , and 2, corresponding to $\zeta=0,1$, and 0 . 
Then, it can be shown that the chirality matrix $\chi=i^{(p-q) / 2} \gamma_{1} \ldots \gamma_{2 \ell}$ anticommutes with all $\gamma_{j}$ and satisfies $\chi^{\dagger}=\chi, \chi^{2}=1$, and $\bar{\chi}=(-1)^{\zeta} \chi$. To construct the indefinite inner products, we define linear operators $\eta_{ \pm}$(called fundamental symmetries) such that $(\phi, \psi)_{ \pm}=\left\langle\phi, \eta_{ \pm} \psi\right\rangle, \eta_{ \pm}^{\dagger}=\eta_{ \pm}$, and $\eta_{ \pm}^{2}=1$. From Eq. (1), $\eta_{ \pm} \gamma_{j}^{\dagger} \eta_{ \pm}= \pm \gamma_{j}$. These fundamental symmetries are unique up to a sign and are built as follows. Let $M_{s}$ be the product of all self-adjoint gamma matrices and $M_{a s}$ be the product of the anti-self-adjoint ones. Then

$$
\begin{aligned}
& \eta_{+}=i^{q(q-1) / 2} \chi^{q} M_{a s}, \\
& \eta_{-}=i^{p(p+1) / 2} \chi^{p} M_{s} .
\end{aligned}
$$

To construct the charge conjugations $J_{ \pm}$, let $n_{i}$ be the number of imaginary gamma matrices and $N$ be their product. Then

$$
\begin{aligned}
& J_{+}=i^{(1-\zeta)(p-q) / 2} \chi^{n_{i}} N K, \\
& J_{-}=i^{(1-\zeta)(p-q) / 2+\zeta} \chi^{n_{i}+1} N K,
\end{aligned}
$$

where $K$ stands for complex conjugation. The relation $\zeta=n_{i}+(p-q) / 2 \bmod 2$ holds. The charge conjugations $J_{ \pm}$satisfy $J_{ \pm} \gamma_{j}= \pm \gamma_{j} J_{ \pm}, J_{ \pm}^{\dagger} J_{ \pm}=1$, and $J_{ \pm}$commutes with $K$. These conditions determine $J_{+}$and $J_{-}$uniquely up to a sign.

In every Clifford algebra $C \ell(p, q)$ with $p+q$ even, we can choose $J=J_{+}$or $J=J_{-}$and $\eta=\eta_{+}$ or $\eta=\eta_{-}$. This makes four possible conventions, which we call cardinal conventions, for which we have

$$
\begin{aligned}
J^{2} & =\epsilon, \\
J \chi & =\epsilon^{\prime \prime} \chi J, \\
J \eta & =\epsilon \kappa \eta J, \\
\eta \chi & =\epsilon^{\prime \prime} \kappa^{\prime \prime} \chi \eta,
\end{aligned}
$$

where $\epsilon=(-1)^{n(n+2) / 8}, \epsilon^{\prime \prime}=(-1)^{n / 2}, \kappa=(-1)^{m(m+2) / 8}$, and $\kappa^{\prime \prime}=(-1)^{m / 2}$, for some integers $m$ and $n$ modulo 8 (see Table I). Note that $\epsilon$ and $\epsilon^{\prime \prime}$ are the same functions of $n$ as $\kappa$ and $\kappa^{\prime \prime}$ are of $m$. These signs were defined so that $\epsilon$ and $\epsilon^{\prime \prime}$ agree with Connes' KO-dimension tables. ${ }^{5,17}$ More precisely, $n$ agrees with Connes' $K O$-dimension, while $m$ is a new dimension, which we call metric dimension.

In physics, the Dirac operator is written as $D=i \gamma^{\mu} \nabla_{\mu}$ in the so-called West-coast convention ${ }^{18}$ and $D=\gamma^{\mu} \nabla_{\mu}$ in the East-coast one. Charge-conjugation symmetry requires $J D=D J$, and the reality of the fermionic Lagrangian implies that $D$ is self-adjoint with respect to the indefinite inner product. Thus, the West-coast convention corresponds to $J=J_{-}$and $\eta=\eta_{+}$, while the East-coast one corresponds to $J=J_{+}$and $\eta=\eta_{-}{ }^{19}$ This is related to the signature of the metric. Indeed, in Minkowski spacetime, we use plane wave solutions of the (massive) Dirac equation $\psi(x)=u e^{i k_{\mu} x^{\mu}}$. Compatibility with the dispersion relation $k^{2}=m_{e}^{2}$, where $m_{e}$ is the fermion mass, implies the metric $(+,-,-,-)$ for the West-coast convention and $(-,+,+,+)$ for the East-coast one. ${ }^{19}$

In Euclidean space, we can be interested in the real solutions $\psi(x)=u e^{k_{\mu} x^{\mu}}$. The metric $(-,-,-$, - ) corresponding to $J=J_{-}$and $\eta=\eta_{-}$is often used, and we call it the North-coast convention because Euclid lived on the coast of North Africa. The remaining possibility is $J=J_{+}$and $\eta=\eta_{+}$that we call the South-coast convention. The values of these dimensions in terms of the cardinal conventions are given in Table II. Related tables can be found in the literature. ${ }^{20-22}$

In Lorentzian spacetime, we need plane waves to describe scattering experiments. Therefore, only the West- and East-coast conventions are allowed. It is interesting to note that, for both conventions, the dimensions $n$ and $m$ are the same. Indeed, in the West-coast convention, $(p, q)=(1,3)$ and

TABLE I. Signs $\epsilon, \kappa$ and $\epsilon^{\prime \prime}, \kappa^{\prime \prime}$ in terms of the metric and $K O$ dimensions $m$ and $n$.

\begin{tabular}{lcccc}
\hline \hline$m, n$ & 0 & 2 & 4 & 6 \\
\hline$\kappa, \epsilon$ & 1 & -1 & -1 & 1 \\
$\kappa^{\prime \prime}, \epsilon^{\prime \prime}$ & 1 & -1 & 1 & -1 \\
\hline \hline
\end{tabular}


TABLE II. Metric, $K O$-dimension $(m, n)$, and charge conjugation, fundamental symmetry $(J, \eta)$ for the four cardinal conventions. The dimensions $m$ and $n$ are to be understood modulo 8 .

\begin{tabular}{lcccc}
\hline \hline Convention & $m$ & $n$ & $J$ & $\eta$ \\
\hline West-coast & $p+q$ & $p-q$ & $J_{-}$ & $\eta_{+}$ \\
East-coast & $p+q$ & $q-p$ & $J_{+}$ & $\eta_{-}$ \\
North-coast & $-p-q$ & $p-q$ & $J_{-}$ & $\eta_{-}$ \\
South-coast & $-p-q$ & $q-p$ & $J_{+}$ & $\eta_{+}$ \\
\hline \hline
\end{tabular}

TABLE III. $(t, s)$ where $t$ is the number of time dimensions and $s$ is the number of space dimensions $s$ as a function of $m$ and $n$. The general solution is $(t+8 j, s+8 k)$, where $j$ and $k$ are integers. The relation between $(p, q)$ and $(t, s)$ is $p=t, q=s$ (West coast); $p=s, q=t$ (East coast); $p=-s, q=-t$ (North coast); and $p=-t, q=-s$ (South coast).

\begin{tabular}{lcccc}
\hline \hline & $n=0$ & 2 & 4 & 6 \\
\hline$m=0$ & $(0,0)(4,4)$ & $(1,7)(5,3)$ & $(2,6)(6,2)$ & $(3,5)(7,1)$ \\
2 & $(1,1)(5,5)$ & $(2,0)(6,4)$ & $(3,7)(7,3)$ & $(0,2)(4,6)$ \\
4 & $(2,2)(6,6)$ & $(3,1)(7,5)$ & $(4,0)(0,4)$ & $(1,3)(5,7)$ \\
6 & $(3,3)(7,7)$ & $(4,2)(0,6)$ & $(5,1)(1,5)$ & $(6,0)(2,4)$ \\
\hline \hline
\end{tabular}

$n=p-q=6 \bmod 8$, while for the East-coast convention $(p, q)=(3,1)$ and $n=q-p=6 \bmod$ 8. In other words, the $K O$ dimension $n$ is the number of time dimensions $t$ minus the number of space dimensions $s$, while $m$ is their sum $t+s$ whatever the convention is. It is therefore tempting to interpret the dimensions $m$ and $n$ in terms of time and space dimensions by solving $n=t-s \bmod 8$ and $m=t+s \bmod 8$. This associates a space dimension and a time dimension with any Clifford algebra.

By inverting the relation between $(s, t)$ and $(m, n)$, we can associate $t w o$ pairs of space and time dimensions $(j, k)$ modulo 8 with every pair $(m, n)$ (see Table III). Indeed, if $(j, k)$ is a solution of $j-k=n \bmod 8$ and $j+k=m \bmod 8$, then $(j+4, k+4)$ is also a solution. This corresponds to the Clifford algebra isomorphism $C \ell(s, t+8) \simeq C \ell(s+8, t) \simeq C \ell(s+4, t+4) .^{23}$ The relation between $(s, t)$ and $(p, q)$ for the four conventions is given in the caption of Table III.

\section{GENERALIZATION}

We generalize the previous results by defining a $C P T$ corepresentation to be a quadruple $S=(\mathcal{H}, \chi, \eta, J)$, where $\mathcal{H}$ is a complex Hilbert space equipped with two self-adjoint involutions $\chi$ and $\eta$ (i.e., $\chi^{\dagger}=\chi, \eta^{\dagger}=\eta$, and $\chi^{2}=\eta^{2}=1$ ) and an anti-unitary operator $J$ that satisfy Eqs. (2)-(5) for some signs $\epsilon, \epsilon^{\prime \prime}, \kappa$, and $\kappa^{\prime \prime}$. We coined the name $C P T$ corepresentation because a representation involving anti-linear operations is called a corepresentation ${ }^{24}$ and because $J, \chi$, and $\eta$ generate the same group as the lift of $C, P$, and $T$ operations to the spinor bundle.

By using the chirality operator $\chi$, we can write $\mathcal{H}=\mathcal{H}_{+} \oplus \mathcal{H}_{-}$, where $\chi v= \pm v$ for $v \in \mathcal{H}_{ \pm}$. An element $v$ of $\mathcal{H}_{ \pm}$is said to be homogeneous, and its parity is $|v|=0$ if $v \in \mathcal{H}_{+}$and $|v|=1$ if $v \in \mathcal{H}_{-}$. The parity of a linear or antilinear map $T$ on $\mathcal{H}$ is $|T|=0$ if $\chi T \chi=T$ and $|T|=1$ if $\chi T \chi=-T$. From relations (3) and (5), we see that $\epsilon^{\prime \prime}=(-1)^{|J|}$ and $\kappa^{\prime \prime}=(-1)^{|\eta|+|J|}$.

The graded tensor product $\hat{\otimes}$ of operators is defined by $\left(T_{1} \hat{\otimes} T_{2}\right)\left(\phi_{1} \otimes \phi_{2}\right)=(-1)^{\left|T_{2}\right|\left|\phi_{1}\right|} T_{1} \phi_{1} \otimes T_{2} \phi_{2}$ when $\phi_{1}$ and $\phi_{2}$ are homogeneous. It is the natural tensor product of Clifford algebras, thanks to Chevalley's relation, ${ }^{25}$

$$
C \ell\left(p_{1}, q_{1}\right) \hat{\otimes} C \ell\left(p_{2}, q_{2}\right)=C \ell\left(p_{1}+p_{2}, q_{1}+q_{2}\right),
$$

which shows that the graded tensor product is indeed compatible with space and time dimensions.

The graded tensor product $T_{1} \hat{\otimes} T_{2}$ is the same operator as the non-graded tensor product $T_{1} \chi_{1}^{\left|T_{2}\right|} \otimes T_{2}$. For example, the graded Dirac operator of the tensor product $D=D_{1} \hat{\otimes} 1+1 \hat{\otimes} D_{2}$ is the same operator as the one given by Connes, $D=D_{1} \otimes 1+\chi_{1} \otimes D_{2}$. 
Let us consider two $C P T$ corepresentations $S_{1}=\left(\mathcal{H}_{1}, \chi_{1}, \eta_{1}, J_{1}\right)$ and $S_{2}=\left(\mathcal{H}_{2}, \chi_{2}, \eta_{2}, J_{2}\right)$ with signs determined by $\left(m_{1}, n_{1}\right)$ and $\left(m_{2}, n_{2}\right)$, respectively. We now want to define a graded tensor product $S_{1} \hat{\otimes} S_{2}=(\mathcal{H}, \chi, \eta, J)$ which is also a $C P T$ corepresentation. We naturally have $\mathcal{H}=\mathcal{H}_{1} \otimes \mathcal{H}_{2}$. We claim that we should define

$$
\begin{aligned}
& \chi=\chi_{1} \hat{\otimes} \chi_{2}, \\
& J=J_{1} \chi_{1}^{\left|J_{2}\right|} \hat{\otimes} J_{2} \chi_{2}^{\left|J_{1}\right|}, \\
& \eta=i^{\left|\eta_{1}\right|\left|\eta_{2}\right|} \eta_{1} \chi_{1}^{\left|\eta_{2}\right|} \hat{\otimes} \eta_{2} \chi_{2}^{\left|\eta_{1}\right|} .
\end{aligned}
$$

These formulas can be derived by treating the case where $\mathcal{H}_{1}$ and $\mathcal{H}_{2}$ are the spinor spaces of two Clifford algebras. If $\left\{\gamma_{i}\right\}$ and $\left\{\gamma_{j}^{\prime}\right\}$ are generators of $C \ell\left(p_{1}, q_{1}\right)$ and $C \ell\left(p_{2}, q_{2}\right)$, respectively, then $\left\{\gamma_{i} \hat{\otimes} 1,1 \hat{\otimes} \gamma_{j}^{\prime}\right\}$ are generators of $C \ell\left(p_{1}+p_{2}, q_{1}+q_{2}\right)$ by the isomorphism (6). It is easily checked that $\chi$ given above is self-adjoint, squares to 1 , and anti-commutes with the gamma matrices $\gamma_{i} \hat{\otimes} 1$ and $1 \hat{\otimes} \gamma_{i}^{\prime}$. We conclude by invoking uniqueness (up to a sign) of the chirality operator. Similarly, if $J_{1}$ and $J_{2}$ are chosen to anti-commute with gamma matrices (so they are $\left(J_{1}\right)_{-}$and $\left.\left(J_{2}\right)_{-}\right)$, then $J$ given by Eq. (8) is an anti-unitary map that defines $J_{-}$up to a phase. Finally, if $\left(\eta_{1}\right)_{+}$and $\left(\eta_{2}\right)_{+}$are inserted into Eq. (9), then $\eta$ is a self-adjoint involution that defines the unique indefinite inner product (up to a sign ${ }^{9}$ ) on $\mathcal{H}$ such that the $\gamma_{i} \hat{\otimes} 1$ and the $1 \hat{\otimes} \gamma_{i}^{\prime}$ are Krein-self-adjoint (see Sec. IV).

The first remarkable property of this tensor product is that it holds for any cardinal convention although we derived it for the West-coast one. In other words, Eq. (8) with $\left(J_{1}\right)_{+}$and $\left(J_{2}\right)_{+}$gives $J_{+}$ and Eq. (9) with $\left(\eta_{1}\right)_{-}$and $\left(\eta_{2}\right)_{-}$gives $\eta_{-}$. The second property is that it can be used to define the tensor product of two $C P T$ corepresentations. In that general case, the tensor product is associative and symmetric, the charge conjugation satisfies

$$
J\left(T_{1} \hat{\otimes} T_{2}\right) J^{-1}=J_{1} T_{1} J_{1}^{-1} \hat{\otimes} J_{2} T_{2} J_{2}^{-1}
$$

for arbitrary linear operators $T_{1}$ and $T_{2}$, and the scalar and Krein products on $\mathcal{H}=\mathcal{H}_{1} \otimes \mathcal{H}_{2}$ are explicitly given by

$$
\left\langle\phi_{1} \otimes \phi_{2}, \psi_{1} \otimes \psi_{2}\right\rangle=\left\langle\phi_{1}, \psi_{1}\right\rangle_{1}\left\langle\phi_{2}, \psi_{2}\right\rangle_{2}
$$

and

$$
\left(\phi_{1} \otimes \phi_{2}, \psi_{1} \otimes \psi_{2}\right)=i^{\left|\eta_{1}\right|\left|\eta_{2}\right|}\left(\phi_{1}, \psi_{1}\right)_{1}\left(\phi_{2}, \chi_{2}^{\left|\eta_{1}\right|} \psi_{2}\right)_{2} .
$$

The first formula is by definition, and the second formula comes from the definition of $\eta$ in Eq. (9). This ensures the Kasparov identities

$$
\begin{aligned}
& \left(T_{1} \hat{\otimes} T_{2}\right)^{\times}=(-1)^{\left|T_{1}\right|\left|T_{2}\right|} T_{1}^{\times} \hat{\otimes} T_{2}^{\times}, \\
& \left(T_{1} \hat{\otimes} T_{2}\right)^{\dagger}=(-1)^{\left|T_{1}\right|\left|T_{2}\right|} T_{1}^{\dagger} \hat{\otimes} T_{2}^{\dagger}
\end{aligned}
$$

for the tensor product of two linear operators and

$$
\begin{aligned}
& \left(T_{1} \hat{\otimes} T_{2}\right)^{\times}=(-1)^{\left|\eta_{1}\right|\left|\eta_{2}\right|+\left|T_{1}\right|\left|T_{2}\right|} T_{1}^{\times} \hat{\otimes} T_{2}^{\times}, \\
& \left(T_{1} \hat{\otimes} T_{2}\right)^{\dagger}=(-1)^{\left|T_{1}\right|\left|T_{2}\right|} T_{1}^{\dagger} \hat{\otimes} T_{2}^{\dagger}
\end{aligned}
$$

for the tensor product of two antilinear operators.

The last remarkable property of the tensor product of $C P T$ corepresentations is that it is additive for the dimension pairs $(m, n)$ and $(s, t)$. It can be checked that $\chi$ and $\eta$ are self-adjoint involutions and $J$ is an anti-unitary map, which satisfy Eqs. (2)-(5) for the signs of some dimensions $(m, n)$. Indeed, we first observe through an explicit calculation that the signs associated with $S_{1} \hat{\otimes} S_{2}$ only depend on the signs associated with $S_{1}$ and $S_{2}: \epsilon=(-1)^{\left|J_{1}\right|\left|J_{2}\right|} \epsilon_{1} \epsilon_{2}$ (where $(-1)^{\left|J_{1}\right|\left|J_{2}\right|}=\left(1+\epsilon_{1}^{\prime \prime}+\right.$ $\left.\left.\epsilon_{2}^{\prime \prime}-\epsilon_{1}^{\prime \prime} \epsilon_{2}^{\prime \prime}\right) / 2\right), \epsilon^{\prime \prime}=\epsilon_{1}^{\prime \prime} \epsilon_{2}^{\prime \prime}, \kappa=(-1)^{\left(\left|\eta_{1}\right|+\left|J_{1}\right|\right)\left(\left|\eta_{2}\right|+\left|J_{2}\right|\right)} \kappa_{1} \kappa_{2}$, and $\kappa^{\prime \prime}=\kappa_{1}^{\prime \prime} \kappa_{2}^{\prime \prime}$. Then, the additivity of the dimensions of $C P T$ corepresentations follows from the fact that it holds for Clifford algebras [see Eq. (6)].

These space and time dimensions can be used to classify topological insulators and superconductors with symmetries, as well as for the investigation of $P T$-symmetric Hamiltonians, because of the presence of a Krein-space structure, ${ }^{26-28}$ to which we now turn because it is crucial to generalize spectral triples to pseudo-Riemannian manifolds. 


\section{KREIN SPACES}

We already introduced Hilbert spaces $\mathcal{H}$ (with a scalar product denoted as $\langle\cdot, \cdot\rangle$ ), which are equipped with a fundamental symmetry (i.e., a self-adjoint operator $\eta$ such that $\eta^{2}=1$ ) defining an indefinite inner product $(\phi, \psi)=\langle\phi, \eta \psi\rangle$. In the mathematical literature, such a space is called a Krein space. Baum ${ }^{8}$ showed that the spinor bundle of a pseudo-Riemannian manifold is naturally equipped with the structure of a Krein space. Her work was extended by Strohmaier to noncommutative geometry. ${ }^{29}$

Substituting an indefinite inner product for a scalar product has a striking physical consequence: the possible existence of states with negative "probabilities" (i.e., such that $(\psi, \psi)<0$ ). These states were first met in physics by Dirac in 1942 in his quantization of electrodynamics. ${ }^{30}$ He interpreted negative-probability states as describing a hypothetical world. ${ }^{31}$ Negative-probability states have now become familiar in physics through their role in the Gupta-Bleuler and Becchi-Rouet-Stora-Tyutin (BRST) quantizations of gauge fields.

In most applications, the indefinite inner product $(\cdot, \cdot)$ is natural (i.e., uniquely defined, up to a scalar factor, by some symmetry conditions) and the scalar product $\langle\cdot, \cdot\rangle$ is somewhat arbitrary. In a Lorentzian manifold, the indefinite inner product is defined by the Lorentzian metric and the scalar product corresponds to the Wick rotation following some choice of a time-like direction (see Ref. 32 for a precise definition). Krein spaces are a natural framework for gauge field theories ${ }^{33-35}$ and Lorentzian spectral triples. ${ }^{7,29,32,36-39}$

We present now some essential properties of operators on Krein spaces. If $\mathcal{K}$ is a Krein space and $T: \mathcal{K} \rightarrow \mathcal{K}$ is a linear operator, its Krein-adjoint $T^{\times}$is defined by $\left(T^{\times} x, y\right)=(x, T y)$ for $x$ and $y$ in $\mathcal{K}$. If $T$ is unbounded, we need to take care of its domain, as described in detail in Ref. 40. A linear operator $T$ is Krein-self-adjoint if $T^{\times}=T$ and Krein-unitary if $T^{\times} T=T T^{\times}=1$.

An anti-linear map (i.e., a map $T: \mathcal{K} \rightarrow \mathcal{K}$ such that $T(\alpha x+\beta y)=\bar{\alpha} T x+\bar{\beta} T y$ ) has a Krein-adjoint $T^{\times}$usually defined by $\left(y, T^{\times} x\right)=(x, T y)$. It is Krein-anti-unitary if, furthermore, $T^{\times} T=T T^{\times}=1$.

Any fundamental symmetry $\eta$ is Krein self-adjoint. The relation between the Krein adjoint $T^{\times}$ and the Hilbert adjoint $T^{\dagger}$ with respect to the scalar product of $\mathcal{H}$ is $T^{\dagger}=\eta T^{\times} \eta$.

In physical applications, the Krein adjoint is the most natural. For example, the Dirac operator on a pseudo-Riemannian manifold is Krein-self-adjoint. In Gupta-Bleuler quantization, the Krein adjoint of a covariant operator is covariant. In gauge field theory, the BRST charge is Krein self-adjoint. The Hilbert adjoint depends on the choice of a fundamental symmetry, in particular it is not covariant in Gupta-Bleuler quantization and the BRST approach. We can now define an indefinite spectral triple.

\section{INDEFINITE SPECTRAL TRIPLE}

After some pioneering studies, ${ }^{41-50}$ many papers were devoted to the extension of noncommutative geometry to Lorentzian geometry. ${ }^{7,29,32,36-38,51-81}$ Inspired by these references, we define an even-dimensional real indefinite spectral triple to be as follows:

1. A $*$-algebra $\mathcal{A}$ represented on a Krein space $\mathcal{K}$ equipped with a Hermitian form $(\cdot, \cdot)$ and a fundamental symmetry $\eta$. We assume that the representation satisfies $\pi\left(a^{*}\right)=\pi(a)^{\times}$.

2. A chirality operator $\chi$, i.e., a linear map on $\mathcal{K}$ such that $\chi^{2}=1$ and $\chi^{\dagger}=\chi$ (where the adjoint $\chi^{\dagger}$ is defined by $\chi^{\dagger}=\eta \chi^{\times} \eta$ ). The algebra commutes with $\chi$.

3. An antilinear charge conjugation $J$ such that $J^{\dagger} J=1$.

4. A set of signs $\left(\epsilon, \epsilon^{\prime \prime}, \kappa, \kappa^{\prime \prime}\right)$ describing relations (2)-(5) between $\chi, \eta$, and $J$.

5. A Krein-self-adjoint Dirac operator $D$, which satisfies $J D=D J$ and $\chi D=-D \chi$.

The name indefinite spectral triple was also used by Van den Dungen and Rennie with a slightly different meaning. ${ }^{74}$ The hypothesis $\pi\left(a^{*}\right)=\pi(a)^{\times}$is a simplifying assumption, which is well adapted to particle physics applications, but which need not be true when discrete structures replace manifolds. ${ }^{82}$ We refer the reader to Refs. $29,32,68$, and 74 for the functional analytic aspects of indefinite spectral triples. If we compare with Connes' spectral triples, we see that we have an additional object 
(the fundamental symmetry $\eta$ ) and two additional signs, $\kappa$ and $\kappa^{\prime \prime}$. Because of this more complex structure, the $K O$-dimension $n$ is no longer enough to classify indefinite spectral triples and we need both $m$ and $n$. The classification carried out in Sec. III holds also for indefinite spectral triples because they are particular cases of $C P T$ corepresentations.

Let $\left(\mathcal{A}_{1}, \mathcal{H}_{1}, D_{1}, J_{1}, \chi_{1}, \eta_{1}\right)$ and $\left(\mathcal{A}_{2}, \mathcal{H}_{2}, D_{2}, J_{2}, \chi_{2}, \eta_{2}\right)$ be two real even-dimensional indefinite spectral triples. If the tensor products defined in Sec. III are supplemented with

$$
\begin{aligned}
\mathcal{A} & =\mathcal{A}_{1} \hat{\otimes} \mathcal{A}_{2}, \\
D & =D_{1} \hat{\otimes} 1+1 \hat{\otimes} D_{2}, \\
\pi & =\pi_{1} \hat{\otimes} \pi_{2},
\end{aligned}
$$

it can be checked that we obtain a real even-dimensional indefinite spectral triple (in particular, $D$ is Krein-self-adjoint, commutes with $J$, and anticommutes with $\chi$ ).

The extension of this tensor product to odd-dimensional indefinite spectral triples seems difficult, if one considers the complexity of the Riemannian case. ${ }^{83-88}$ Note that Farnsworth also advocated the use of a graded tensor product. ${ }^{87}$

We define now the indefinite spectral triple encoding models of particle physics.

\section{PARTICLE PHYSICS MODELS}

Particle physics models (QED, electroweak, standard model) are described by an almost commutative spectral triple, i.e., the tensor product of the spectral triple $\mathcal{S}_{1}$ of a manifold $M$ and a finite dimensional spectral triple $\mathcal{S}_{2}$.

Connes and Lott $^{89}$ derived the fermionic and gauge Lagrangians of the standard model in Riemannian space. In Lorentzian spacetime, Van den Dungen found the fermionic Lagrangian, but the gauge Lagrangian is considered to be an open problem. ${ }^{7}$ Surprisingly, the problem was already solved in Elsner's outstanding M.S. thesis ${ }^{47}$ (see also Refs. 90-92), where many aspects of noncommutative geometry (e.g., Connes differential algebra, curvature, bosonic and fermionic Lagrangians) are generalized to the case where the Hilbert space is replaced by a vector space equipped with a sesquilinear form. Indefinite spectral triples clearly fit into that framework. When the base manifold $M$ is not compact, Elsner defines a family of bosonic Lagrangians $A_{n}=\int_{U_{n}} \sqrt{|g|} d x \mathcal{L}_{b}(x)$, where the relatively compact open sets $U_{n}$ form an exhausting family covering $M$ and $\mathcal{L}_{b}=-\operatorname{Re} \operatorname{Tr}\left(\theta^{\times} \theta\right)$ is the Lagrangian density. The real part is required because the algebra is real. ${ }^{47}$ The two-form $\theta$ in Connes' differential algebra is the curvature of the gauge potential $\rho$, and the trace is

$$
\operatorname{Tr}\left(M_{1} \otimes M_{2}\right)=\operatorname{Tr}_{1}\left(M_{1}\right) \operatorname{Tr}_{2}\left(M_{2}\right),
$$

where $\operatorname{Tr}_{1}$ is the trace over the spinor fibre over $M$ and $\operatorname{Tr}_{2}$ is the trace over the finite dimensional Krein space $\mathcal{K}_{2}$.

The family of open sets ensures that, for any compactly supported variation, there is an $n_{0}$ such that $U_{n}$ contains the support of the variation for every $n>n_{0}$ and the variation of $A_{n}$ is well defined. This is compatible with the NCG point of view because Van Suijlekom proved the conceptually important fact that any noncommutative geometry can be considered as an algebra bundle over a Hausdorff base space. ${ }^{93}$ We calculate $\mathcal{L}_{b}$ for QED in this paper and for the standard model in a forthcoming publication. We use the fermionic Lagrangian $\mathcal{L}_{f}=\left(\Psi,\left(D+A+J A J^{-1}\right) \Psi\right){ }^{7,47,52}$ Note that in the Riemannian NCG Lagrangian the first $\Psi$ is replaced by $J \Psi .{ }^{17}$

An important and long-standing problem is that only physical states $\Psi$ must be used in the fermionic Lagrangian, while the trace $\operatorname{Tr}$ is over the whole space $\mathcal{K}_{1} \otimes \mathcal{K}_{2}$. Lizzi et al. ${ }^{43}$ pointed out that their bosonic Lagrangian over physical states is different from $\mathcal{L}_{b}$ and not physically valid (it contains $C P T$-symmetry violating terms). We discuss now this so-called fermion doubling problem.

\section{FERMION DOUBLING PROBLEM}

In this section, we consider a four-dimensional Lorentzian manifold $M$ and the finite dimensional space is written as the sum $\mathcal{K}_{2}=\mathcal{K}_{L} \oplus \mathcal{K}_{R} \oplus \mathcal{K}_{\bar{R}} \oplus \mathcal{K}_{\bar{L}}$ of left and right particles and their 
antiparticles. A pseudo-orthonormal basis of these spaces is denoted by $p_{L}^{j}, p_{R}^{j}, p_{R}^{j c}=J_{2} p_{R}^{j}$, and $p_{L}^{j c}=J_{2} p_{L}^{j}$, respectively. In QED $j=1$, because the electron is the only particle, in the standard model $j=1, \ldots, 24$ (three generations of neutrinos, electrons, up and down quarks with three colors). The name of the fermion doubling (or quadrupling) problem comes from the fact that a state in $\mathcal{K}_{1} \otimes \mathcal{K}_{2}$ corresponding to a particle $p$ can be written as a linear combination of $\psi \otimes p_{L}$, $\psi \otimes p_{R}, \psi \otimes p_{L}^{c}$, and $\psi \otimes p_{R}^{c}$. Since $\psi \in \mathcal{K}_{1}=\Gamma(M, S)$ is a four-dimensional (Dirac) spinor, each particle with its antiparticle is described by a 16-dimensional vector instead of a four-dimensional one. It was also observed that the fermion doubling problem is different in Lorentzian and Riemannian signatures. ${ }^{94}$

To solve the problem, we call $V_{\text {phys }}$ the set of states of the form $\Psi=(1+J) \Psi_{0}$, where

$$
\Psi_{0}=\sum_{j} \psi_{L}^{j} \otimes p_{L}^{j}+\psi_{R}^{j} \otimes p_{R}^{j}
$$

where $\psi_{L}^{j}$ and $\psi_{R}^{j}$ are two-dimensional Weyl fermions (obtained as $\chi_{1}^{ \pm} \psi$ where $\chi_{1}^{ \pm}=\left(1 \pm \chi_{1}\right) / 2$ ). This solution is a variation of those proposed by Lizzi et al. ${ }^{43}$ and Elsner et al. ${ }^{47,92}$

The degrees of freedom are reduced to four for each particle (two for $\psi_{L}^{j} \otimes p_{L}^{j}$ and two for $\psi_{R}^{j} \otimes p_{R}^{j}$ ). The antiparticle states do not correspond to additional degrees of freedom because they are obtained by applying $J$ to the particle states. ${ }^{47,95}$ These physical states can be singled out by the requirement that they are invariant under all Krein-unitary operators commuting with $J$ and $\chi$. They satisfy the Weyl condition $\Psi=\chi \Psi^{96}$ and the Majorana condition $\Psi=J \Psi$, first proposed by Barrett ${ }^{52}$ (when $J^{2}$ $=1$ ). If one requires only invariance under the gauge group and local Lorentz transformations, more general physical states are possible.

Lizzi et al. ${ }^{43}$ noticed that left-handed particles (for the Lorentz group) $\psi_{L}$ must also be lefthanded particles $p_{L}$ for the gauge group to be physically meaningful. In Grand Unified Theories (GUT), where each particle $p$ also appears in four varieties: $p_{L}, p_{R}, p_{L}^{c}$, and $p_{R}^{c},{ }^{97}$ the identification of the spinor and gauge variables is so obvious that it is implicit and a state like $\psi_{L} \otimes p_{L}$ is simply denoted by $p_{L}$.

We can now define the trace of $M=M_{1} \otimes M_{2}$ over physical states as

$$
\operatorname{Tr}^{\prime} M=\sum_{k, j, P=L / R}\left\langle(1+J) \Psi_{P}^{k j},\left(M_{1} \otimes M_{2}\right)(1+J) \Psi_{P}^{k j}\right\rangle,
$$

where $\Psi_{P}^{k j}=e_{P}^{k} \otimes p_{P}^{j}$ and $e_{P}^{k}$ is a basis of two-component spinors $\psi_{P}$. Note that $\operatorname{Tr}$ and $\operatorname{Tr}^{\prime}$ are different because Tr has sums over states which are not in $V_{\text {phys }}$, such as $\psi_{L} \otimes p_{R}$.

This choice of physical states solves the fermion doubling problem because, although $\operatorname{Tr}$ and $\operatorname{Tr}^{\prime}$ are generally not proportional, $\operatorname{Re} \operatorname{Tr} \theta^{\times} \theta=2 \operatorname{Re} \operatorname{Tr}^{\prime} \theta^{\times} \theta$ for the indefinite spectral triples of QED and the standard model. This will be clear for QED and will be discussed for the standard model in a forthcoming paper.

We can restate this result as follows. We have two natural indefinite inner products on the space of operators. The first one, $(A, B)=\operatorname{Re} \operatorname{Tr}\left(A^{\times} B\right)$, is determined by the invariance under Krein-unitary transformations, and the second one, $(A, B)^{\prime}=\operatorname{Re} \operatorname{Tr}^{\prime}\left(A^{\times} B\right)$, is determined by the set $V_{\text {phys }}$ of physical states. It is important to check that both properties are consistent for physical models.

\section{INDEFINITE SPECTRAL TRIPLE OF QED}

The NCG model of QED in Riemannian spacetime was described by Van den Dungen and Van Suijlekom ${ }^{98}$ and in Lorentzian space by Van den Dungen, ${ }^{7}$ who did not include antiparticles and the charge conjugation operator. The manifold spectral triple consists of a Lorentzian 4D manifold $M$, a Dirac operator $D_{1}=i \gamma^{\mu} \nabla_{\mu}$, where $\nabla_{\mu}$ is the covariant derivative (including the spin connection), and the Krein space $\Gamma(M, S) .{ }^{8}$ In the chiral representation of the gamma matrices, ${ }^{16}$ the operators are 


$$
\chi_{1}=\left(\begin{array}{cc}
-1 & 0 \\
0 & 1
\end{array}\right), \eta_{1}=\left(\begin{array}{ll}
0 & 1 \\
1 & 0
\end{array}\right), J_{1}=i\left(\begin{array}{cc}
0 & \sigma^{2} \\
-\sigma^{2} & 0
\end{array}\right) K,
$$

where $K$ means the complex conjugation and $\sigma^{2}$ is the Pauli matrix. We use the same algebra $\mathcal{A}=\mathbb{C} \oplus \mathbb{C}$ as van den Dungen ${ }^{7}$ and the Krein space $\mathcal{K}_{2}=\mathbb{C}^{4}$ with basis states $\left(e_{L}, e_{R}, e_{R}^{c}, e_{L}^{c}\right)$. The representation of $(a, b) \in \mathcal{A}$ and the operators are, in terms of Pauli matrix $\sigma^{1}, \sigma^{2}$, and $\sigma^{3}$,

$$
\begin{aligned}
\pi_{2}(a, b) & =\left(\begin{array}{rr}
a 1 & 0 \\
0 & b 1
\end{array}\right), \quad \chi_{2}=-\left(\begin{array}{rr}
\sigma^{3} & 0 \\
0 & \sigma^{3}
\end{array}\right), \\
\eta_{2} & =\left(\begin{array}{rr}
-\sigma^{3} & 0 \\
0 & \sigma^{3}
\end{array}\right), \quad J_{2}=\left(\begin{array}{rr}
0 & \epsilon_{2} \sigma^{1} \\
\sigma^{1} & 0
\end{array}\right) K, \\
\varpi & =\left(\begin{array}{rr}
1 & 0 \\
0 & -1
\end{array}\right), \quad D_{2}=i m\left(\begin{array}{rr}
-\sigma^{2} & 0 \\
0 & \sigma^{2}
\end{array}\right) .
\end{aligned}
$$

To compare the fermionic Lagrangian on particles and antiparticles, we use the relation $(J \Psi, D J \Psi)=\epsilon K(\Psi, D \Psi)$. We determine $\epsilon K$ by computing the dimensions of the total spectral triple. For the manifold spectral triple, $\left(m_{1}, n_{1}\right)=(4,6)$ because its $K O$ dimension $p-q \bmod 6$ is $n_{1}=6^{47}$ and its metric dimension $p+q$ is 4 . For the finite spectral triple, $\left(m_{2}, n_{2}\right)=(2,2)$ if $\epsilon_{2}=-1$ and $\left(m_{2}, n_{2}\right)=(6,6)$ if $\epsilon_{2}=1$. In the literature, it is generally assumed that $n_{2}=6$, but $n_{2}=2$ is also valid for QED (we shall see that $n_{2}=2$ is required for the standard model). The total spectral triple has now dimensions $(m, n)=(6,0)$ if $\epsilon_{2}=-1$ and $(m, n)=(2,4)$ if $\epsilon_{2}=1$. In both cases, $\epsilon \kappa=1$, which implies $(J \Psi, D J \Psi)=(\Psi, D \Psi)$. Thus, we can also define the fermionic Lagrangian over the particle states only.

The physical Lagrangian for QED with a massive electron is

$$
\mathcal{L}=-\frac{1}{4} F^{\mu v} F_{\mu v}+\left(\psi,\left(i \gamma^{\mu}\left(\nabla_{\mu}+i q A_{\mu}\right)-m\right) \psi\right)
$$

where $q<0$ is the electron charge and $F_{\mu \nu}=\partial_{\mu} A_{\nu}-\partial_{\nu} A_{\mu}$. Note that our notation $\left(\psi, \psi^{\prime}\right)$ is usually written as $\bar{\psi} \psi^{\prime}$ in the physics literature.

The Dirac operator corresponding to this triple is

$$
D(A)=D-q \gamma^{\mu} A_{\mu} \otimes \varpi,
$$

where

$$
-q A_{\mu}=i \sum_{j}\left(\pi\left(a_{j}\right) \partial_{\mu} \pi\left(b_{j}\right)+J_{2} \pi\left(a_{j}\right) \partial_{\mu} \pi\left(b_{j}\right) J_{2}^{-1}\right)
$$

is self-adjoint. The curvature is

$$
\theta=-\frac{i q}{2} \sum_{\mu \nu}\left(\gamma^{\mu} \gamma^{\nu}-g^{\mu \nu}\right) F_{\mu \nu} \otimes \varpi,
$$

and the bosonic Lagrangian is

$$
\mathcal{L}_{b}=-8 q^{2} F_{\mu \nu} F^{\mu \nu} .
$$

The prefactor of $\mathcal{L}_{b}$ is not correct. This problem was elegantly solved ${ }^{47,90,99}$ by choosing a positive definite $z$ which commutes with $\pi(\mathcal{A}), J \pi(\mathcal{A}) J^{-1}$, and $D$ to redefine the trace as $\operatorname{Tr}_{z}(M)=\operatorname{Tr}(z M)$. In the case of QED, a solution of the constraints is $z=\rho 1 \otimes 1$, where $\rho>0$. We can now define the Lagrangian

$$
\mathcal{L}_{\mathrm{CLE}}=\operatorname{Re} \operatorname{Tr}_{z}\left(\theta^{\times} \theta\right)+\frac{1}{2}(\Psi, D(A) \Psi),
$$

which we call the Connes-Lott-Elsner (CLE) Lagrangian since it was originally proposed by Connes and Lott in the Riemannian case and then extended by Elsner to general signatures. Observe that, since noncommutative 2-forms are defined modulo the junk, ${ }^{100}$ the expression $\operatorname{Tr}_{z}\left(\theta^{\times} \theta\right)$ does not have an immediate meaning. In the Riemannian case, this problem is solved by projecting $\theta$ onto the orthogonal of the junk. This solution can be applied in general signature if and only if the restriction 
of the indefinite inner product to the junk is non-degenerate. Remarkably, this property holds in QED as well as in the standard model. ${ }^{47,101}$

The QED bosonic Lagrangian becomes $\mathcal{L}_{b}=-8 \rho q^{2} F_{\mu \nu} F^{\mu \nu}$, and we can choose $\rho=1 /\left(32 q^{2}\right)$ to obtain the physical bosonic Lagrangian. The fermionic Lagrangian $\mathcal{L}_{f}=\frac{1}{2}(\Psi, D(A) \Psi)$ with

$$
\Psi=(1+J)\left(\chi_{1}^{+} \psi \otimes e_{R}+\chi_{1}^{-} \psi \otimes e_{L}\right)
$$

becomes

$$
\mathcal{L}_{f}=\left(\psi,\left(i \gamma^{\mu}\left(\nabla_{\mu}+i q A_{\mu}\right)-m\right) \psi\right),
$$

and the CLE-Lagrangian exactly coincides with the physical one in Lorentzian spacetime.

To complete this section, we remark that particles and antiparticles have the same mass and opposite currents. In textbook QED, these properties are achieved through the anticommutativity of the normal product of fermion operators. ${ }^{16}$ In our framework, this property can follow from the fundamental symmetry. Indeed, by using $J_{1} \gamma^{\mu}=-\gamma^{\mu} J_{1}$ and $\left(\gamma^{\mu}\right)^{\times}=\gamma^{\mu}$, we get

$$
\begin{aligned}
\langle J \Psi, \eta J \Psi\rangle & =\langle\Psi, \eta \Psi\rangle, \\
\left\langle J \Psi, \eta\left(\gamma^{\mu} \otimes 1\right) J \Psi\right\rangle & =-\left\langle\Psi, \eta\left(\gamma^{\mu} \otimes 1\right) \Psi\right\rangle,
\end{aligned}
$$

which means that the mass is conserved and the current is reversed by charge conjugation. We did not use any anticommutation relation.

\section{CONCLUSION}

A particularly appealing aspect of noncommutative geometry is that the internal (fibre) and external (manifold) degrees of freedom are put into a common geometric framework. Real Clifford algebras can also unify spacetimes and finite objects since they describe spinors on pseudo-Riemannian manifolds as well as finite geometries. ${ }^{102}$ Therefore, it is not surprising that real Clifford algebras can be used to define the space and time dimensions of an algebra representing (in a generalized sense) a possibly noncommutative spacetime. The present paper is a precise formulation of this idea, and the main ingredient of the definition of a time dimension is the fundamental symmetry $\eta$ which allows for a kind of Wick rotation of spacetime.

The Lorentzian distance has now received a satisfactory noncommutative geometric interpretation, ${ }^{53,79,103-108}$ and an index theorem was proved for Lorentzian manifolds. ${ }^{109}$

What remains to be done to get a complete Lorentzian noncommutative geometry is to find a Lorentzian analog of the spectral action giving the Lagrangian of gauge fields coupled with gravity. The fermionic and gauge Lagrangian of particle physics models were given here for Lorentzian spacetimes, but the derivation of the full spectral action is still a difficult open problem.

For applications to topological insulators, it would be desirable to extend these results to the case of odd-dimensional algebras. An interpretation of CPT corepresentations in the context of twisted equivariant topological K-theory was recently given by Gomi (private communication).

\section{ACKNOWLEDGMENTS}

We are extremely grateful to Harald Upmeier for sending us a copy of Elsner's Master's thesis. We thank Nicolas Franco for sharing with us his extended knowledge of the literature. We thank Shane Farnsworth, Johannes Kellendonk, Guo Chuan Thiang, Karen Elsner, Franciscus Jozef Vanhecke, Bertfried Fauser, Jordan François, Mairi Sakellariadou, Fedele Lizzi, Kiyonori Gomi, Giuseppe De Nittis, Koen van den Dungen, Thomas Schücker, and Christian Bär for discussion and correspondence. We thank the anonymous referee for his or her very stimulating questions and remarks.

\footnotetext{
${ }^{1}$ T. Morimoto and A. Furusaki, Phys. Rev. B 88, 125129 (2013).

${ }^{2}$ C.-K. Chiu, J. C. Y. Teo, A. P. Schnyder, and S. Ryu, Rev. Mod. Phys. 88, 035005 (2016).

${ }^{3}$ M. F. Atiyah, R. Bott, and A. Shapiro, Topology 3(Suppl. 1), 3 (1964).

${ }^{4}$ M. Karoubi, K-Theory: An Introduction (Springer, Berlin, 2008).

${ }^{5}$ A. Connes, J. Math. Phys. 36, 6194 (1995).

${ }^{6}$ B. A. Bernevig and T. L. Hugues, Topological Insulators and Topological Superconductors (Princeton University Press, Princeton, 2013).
} 
${ }^{7}$ K. van den Dungen, Math. Phys. Anal. Geom. 19, 4 (2016).

${ }^{8}$ H. Baum, Spin-Strukturen und Dirac-Operatoren über pseudoriemannschen Mannigfaltigkeiten (Teubner, Lepzig, 1981).

${ }^{9}$ P. L. Robinson, Glasgow Math. J. 30, 263 (1988).

${ }^{10}$ F. R. Harvey, Spinors and Calibrations (Academic Press, Boston, 1990).

${ }^{11}$ W. Pauli, Ann. Inst. Henri Poincaré 6, 109 (1936), available at http://www.numdam.org/item?id=AIHP_1936_6_2_109_0.

12 T. Kugo and P. Townsend, Nucl. Phys. B 221, 357 (1983).

${ }^{13}$ C. Wetterich, Nucl. Phys. B 211, 177 (1983).

${ }^{14}$ M. A. de Andrade, M. Rojas, and F. Toppan, Int. J. Mod. Phys. A 16, 4453 (2001).

${ }^{15}$ D. S. Shirokov, Theor. Math. Phys. 175, 454 (2013).

${ }^{16}$ C. Itzykson and J.-B. Zuber, Quantum Field Theory (McGraw-Hill, New York, 1980).

${ }^{17}$ A. Connes and M. Marcolli, Noncommutative Geometry, Quantum Fields and Motives (American Mathematical Soc., Providence, 2008).

${ }^{18}$ M. J. Duff and J. Kalkkinen, Nucl. Phys. B 758, 161 (2006).

${ }^{19}$ M. Berg, C. Dewitt-Morette, S. Gwo, and E. Kramer, Rev. Math. Phys. 13, 953 (2001).

${ }^{20}$ V. V. Varlamov, Adv. Appl. Clifford Algebr. 25, 487 (2015).

${ }^{21}$ M. Budinich, J. Math. Phys. 57, 071703 (2016).

${ }^{22}$ H. Schulz-Baldes and C. Villegas-Blas, Math. Nachr. 290, 1840 (2017).

${ }^{23}$ D. J. H. Garling, Clifford Algebras: An Introduction, Volume 78 of London Mathematical Society Student Texts (Cambridge University Press, Cambridge, 2011).

${ }^{24}$ E. Wigner, Group Theory (Academic Press, New York, 1959).

${ }^{25}$ H. B. Lawson, Jr. and M.-L. Michelsohn, Spin Geometry (Princeton University Press, Princeton, 1989).

${ }^{26}$ A. Mostafazadeh, J. Math. Phys. 43, 205 (2002).

${ }^{27}$ T. Tanaka, J. Phys. A: Math. Gen. 39, 14175 (2006).

${ }^{28}$ P. D. Manneheim, Phys. Lett. B 753, 288 (2016).

${ }^{29}$ A. Strohmaier, J. Geom. Phys. 56, 175 (2006).

${ }^{30}$ P. A. M. Dirac, Proc. R. Soc. A 180, 1 (1942).

${ }^{31}$ H. Kragh, Dirac a Scientific Biography (Cambridge University Press, Cambridge, 1990).

${ }^{32}$ K. van den Dungen, M. Paschke, and A. Rennie, J. Geom. Phys. 73, 37 (2013).

${ }^{33}$ S. S. Horuzhy and A. V. Voronin, Commun. Math. Phys. 123, 677 (1989).

${ }^{34}$ N. Nakanishi and I. Ojima, Covariant Operator Formalism of Gauge Theories and Quantum Gravity (World Scientific, Singapore, 1990).

${ }^{35}$ F. Strocchi, An Introduction to Non-Perturbative Foundations of Quantum Field Theory (Oxford Science Publications, Oxford, 2013).

${ }^{36}$ W. van Suijlekom, J. Math. Phys. 45, 537 (2004).

${ }^{37}$ M. Paschke and A. Sitarz, "Equivariant Lorentzian spectral triples,” e-print arXiv:math-ph/0611029 (2006).

${ }^{38}$ M. Marcolli, Comm. Numb. Theor. Phys. 2, 421 (2008).

${ }^{39}$ F. Besnard and N. Bizi, J. Geom. Phys. 123, 292 (2018).

${ }^{40}$ J. Bognár, Indefinite Inner Product Spaces (Springer, Berlin, 1974).

${ }^{41}$ W. Kalau, J. Geom. Phys. 18, 349 (1996).

${ }^{42}$ E. Hawkins, Commun. Math. Phys. 187, 471 (1997).

${ }^{43}$ F. Lizzi, G. Mangano, G. Miele, and G. Sparano, Phys. Rev. D 55, 6357 (1997).

44 J. M. Gracia-Bondía, B. Iochum, and T. Schücker, Phys. Lett. B 416, 123 (1997).

${ }^{45}$ T. Kopf, Int. J. Mod. Phys. A 13, 2693 (1998).

${ }^{46}$ C. P. Martín, J. M. Gracia-Bondía, and J. Várilly, Phys. Rep. 294, 363 (1998).

${ }^{47} \mathrm{~K}$. Elsner, Elektroschwaches Modell und Standardmodell in der nichtkommutativen Geometrie (Diplomarbeit, Philipps Universität Marburg, 1999).

${ }^{48}$ T. Kopf, Int. J. Mod. Phys. B 14, 2359 (2000).

${ }^{49}$ T. Kopf and M. Paschke, Mod. Phys. Lett. A 16, 291 (2001).

${ }^{50}$ T. Kopf and M. Paschke, J. Math. Phys. 43, 818 (2002).

${ }^{51}$ P. Bieliavsky, M. Rooman, and P. Spindel, Nucl. Phys. B 645, 349 (2002).

52 J. W. Barrett, J. Math. Phys. 48, 012303 (2007).

${ }^{53}$ V. Moretti, Rev. Math. Phys. 15, 1171 (2003).

${ }^{54}$ M. Paschke and R. Verch, Classical Quantum Gravity 21, 5299 (2004).

${ }^{55}$ P. Bieliavsky, S. Detournay, P. Spindel, and M. Rooman, J. High Energy Phys. 2004(06), 031.

${ }^{56}$ P. Bieliavsky, S. Detournay, P. Spindel, and M. Rooman, in Noncommutative Geometry and Physics, Lecture Notes in Pure and Applied Mathematics, edited by Y. Maeda, N. Tose, N. Miyazaki, S. Watamura, and D. Sternheimer (World Scientific, New Jersey, 2005), pp. 17-33.

${ }^{57}$ T. Kopf, in Fundamental Interactions: Proceedings of the 21 th Lake Louise Winter Institute, edited by A. Astbury, F. Khanna, and R. Moore (2006), Vol. 21, pp. 240-243.

${ }^{58}$ J.-H. Jureit, T. Krajewski, T. Schücker, and C. A. Stephan, Acta Phys. Pol. B 38, 3181 (2007).

${ }^{59}$ M. Paschke, in Quantum Gravity, edited by B. Fauser, J. Tolksdorf, and E. Zeidler (Birkhäuser, Basel, 2007), pp. 127-150.

${ }^{60}$ P. Bieliavsky, L. Claessens, D. Sternheimer, and Y. Voglaire, in Poisson Geometry in Mathematics and Physics, Volume 450 of Contemporary Mathematics, edited by G. Ditto, J.-H. Lu, Y. Maeda, and A. Weinstein (American Mathematical Soc., Providence, 2008), pp. 1-24.

${ }^{61}$ F. Besnard, J. Geom. Phys. 59, 861 (2009).

${ }^{62}$ M. Borris and R. Verch, Commun. Math. Phys. 293, 399 (2010).

${ }^{63}$ W. Nelson and M. Sakellariadou, Phys. Rev. D 81, 085038 (2010).

${ }^{64}$ R. Verch, Acta Phys. Pol. B Proc. Suppl. 4, 507 (2011). 
${ }^{65}$ C. Estrada and M. Marcolli, Int. J. Geom. Methods Mod. Phys. 10, 1250086 (2013).

${ }^{66}$ N. Franco and M. Eckstein, Classical Quantum Gravity 30, 135007 (2013).

${ }^{67}$ N. Franco and M. Eckstein, in Mathematical Structures of the Universe, edited by M. H. M. Eckstein and S. Szybka (Copernicus Center Press, 2014), pp. 315-340.

${ }^{68}$ N. Franco, Rev. Math. Phys. 26, 1430007 (2014).

${ }^{69}$ N. Franco and M. Eckstein, Symmetry, Integrability Geom.: Methods Appl. 10, 010 (2014).

${ }^{70}$ M. Eckstein and N. Franco, in Proceedings of the Conference on Frontiers of Fundamental Physics 14, Proceedings of Science, Vol. FFP14, edited by E. Kajfasz, T. Masson, and R. Triay (SISSA, Trieste, 2015), p. 138.

${ }^{71}$ F. Besnard, J. Phys.: Conf. Ser. 634, 012009 (2015).

${ }^{72}$ N. Franco and M. Eckstein, J. Geom. Phys. 96, 42 (2015).

${ }^{73}$ F. D’Andrea, M. A. Kurkov, and F. Lizzi, Phys. Rev. D 94, 025030 (2016).

${ }^{74}$ K. van den Dungen and A. Rennie, Ann. Henri Poincaré 17, 3255 (2016).

${ }^{75}$ M. Eckstein, N. Franco, and T. Miller, Phys. Rev. D 95, 061701(R) (2017).

${ }^{76}$ N. Franco and J.-C. Wallet, in Noncommutative Geometry and Optimal Transport, Volume 676 of Contemporary Mathematics, edited by P. Martinetti and J.-C. Wallet (American Mathematical Soc., 2016), pp. 147-173.

${ }^{77}$ A. Watcharangkool and M. Sakellariadou, Phys. Rev. D 95, 025027 (2017).

${ }^{78}$ A. Devastato, S. Farnsworth, F. Lizzi, and P. Martinetti, J. High Energy Phys. 03, 089 (2018).

${ }^{79}$ N. Franco, J. Phys.: Conf. Ser. 968, 012005 (2018).

${ }^{80}$ N. Franco, in Proceedings of the 6th International Conference on New Frontiers in Physics, preprint arXiv:1711.05057 (2018).

${ }^{81}$ K. van den Dungen, e-print arXiv:1711.07299.

${ }^{82} \mathrm{~F}$. Besnard, e-print arXiv:1611.07842.

${ }^{83}$ F. J. Vanhecke, Lett. Math. Phys. 50, 157 (1999).

${ }^{84}$ L. Da̧rowski and G. Dossena, Inter. J. Geom. Methods Mod. Phys. 8, 1833 (2011).

${ }^{85}$ F. J. Vanhecke, A. R. da Silva, and C. Sigaud, Braz. J. Phys. 42, 471 (2012).

${ }^{86}$ B. Ćaćić, Lett. Math. Phys. 103, 793 (2013).

${ }^{87}$ S. Farnsworth, J. Math. Phys. 58, 023507 (2017).

${ }^{88}$ S. Guin, e-print arXiv:1712.00986.

${ }^{89}$ A. Connes and J. Lott, Nucl. Phys. B, Proc. Suppl. 18, 29 (1990).

${ }^{90}$ K. Elsner, Modern Phys. Lett. A 16, 241 (2001).

${ }^{91}$ K. Elsner, H. Neumann, and H. Upmeier, in Noncommutative Geometry and the Standard Model of Elementary Particles, Volume 596 of Lecture Notes in Physics, edited by F. Scheck, H. Upmeier, and W. Werner (Springer, Berlin, 2002), pp. 152-171.

${ }^{92}$ K. Elsner, H. Neumann, and H. Upmeier, in Noncommutative Geometry and the Standard Model of Elementary Particles, Volume 596 of Lecture Notes in Physics, edited by F. Scheck, H. Upmeier, and W. Werner (Springer, Berlin, 2002), pp. 172-217.

${ }^{93}$ W. D. van Suijlekom, Adv. Math. 290, 682 (2016)

${ }^{94}$ M. A. Kurkov, F. Lizzi, and D. Vassilevich, Phys. Lett. B 731, 311 (2014).

${ }^{95}$ S. Lazzarini and T. Schücker, Phys. Lett. B 510, 277 (2001).

${ }^{96}$ A. Connes, in Séminaire N. Bourbaki, 1995-1996, Exposé No. 816 (Société Mathématique de France, 1996), pp. 313-349.

${ }^{97}$ J. Baez and J. Huerta, Bull. Am. Math. Soc. 47, 483 (2010).

${ }^{98}$ K. van den Dungen and W. van Suijlekom, J. Noncommutative Geom. 7, 433 (2013).

${ }^{99}$ B. Iochum, D. Kastler, and T. Schücker, J. Math. Phys. 36, 6232 (1995).

${ }^{100}$ A. Connes, Noncommutative Geometry (Academic Press, San Diego, 1994).

${ }^{101}$ N. Bizi, Ph.D. thesis, Sorbonne Université, 2018.

102 R. Shaw, J. Math. Phys. 30, 1971 (1989).

103 G. N. Parfionov and R. R. Zapatrin, J. Math. Phys. 41, 7122 (2000).

${ }^{104}$ N. Franco, Symmetry, Integrability Geom.: Methods Appl. 6, 064 (2010).

105 A. Rennie and B. E. Whale, J. Geom. Phys. 106, 108 (2016).

106 A. Rennie and B. E. Whale, J. Geom. Phys. 117, 277 (2017).

${ }^{107}$ E. Minguzzi, e-print arXiv: 1709.06494.

${ }^{108}$ E. Minguzzi, J. Phys.: Conf. Ser. 968, 012009 (2018).

${ }^{109}$ C. Bär and A. Strohmaier, Commun. Math. Phys. 347, 703 (2016). 